\title{
Pressure Sensing Based on Ratiometric Bragg Grating Loss in a Planar Silica Diaphragm Platform
}

\author{
A. Jantzen ${ }^{1}$ *, P. C. Gow ${ }^{1}$, A. C. Gray ${ }^{1}$, S. L. Scholl ${ }^{1}$, J. C. Gates ${ }^{1}$, P. G. R. Smith ${ }^{1}$, L. J. Boyd ${ }^{2}$, C. Holmes ${ }^{1}$ \\ ${ }^{I}$ Optoelectronics Research Centre, University of Southampton, Southampton, SO17 1BJ, UK \\ ${ }^{2}$ Parker Aerospace, Parker Hannifin Corporation, Bristol, BS32 4AQ \\ *A.Jantzen@soton.ac.uk
}

\begin{abstract}
In this paper the first demonstration of a planar silica-on-silicon chip pressure sensor based on monitoring ratiometric Bragg grating amplitude loss across a diaphragm is made. The device resolution was found to be $0.46 \mathrm{kPa}$.

OCIS codes: (280.4788) Optical sensing and sensors; (280.5475) Pressure measurement; (230.1480) Bragg reflectors
\end{abstract}

\section{Introduction}

Recent pursuits in sectors such as aerospace are increasingly pushing sensor performance by placing them into harsh environments. To address this, optics is seen as an encouraging way of monitoring in electromagnetically noisy environments and areas where spark hazards may be a concern. This work was done in collaboration with Parker Aerospace and to the best of the authors' knowledge, constitutes the first demonstration of a pressure sensor based on Bragg grating reflection-loss in a planar waveguide diaphragm configuration.

To lower the cost and increase the functionality of optical devices, a planar diaphragm chip approach has been developed that is capable of being scaled to wafer level production. Through a series of waveguides and gratings, it has previously been shown that this type of device can be used to monitor pressure by observing the spectral shift of gratings [1]. Noteworthy to the work presented here is a configuration wherein only amplitude changes are noticeable, with no significant spectral shift in Bragg wavelength following an increase in pressure. This alternative approach is a result of extreme residual stress from fabrication causing light to be coupled out of the waveguide more readily as it traverses the bent diaphragm. Comparing the intensity of Bragg gratings before, on, and after the diaphragm, a self-referenced integrated optical device capable of monitoring pressure is realized.

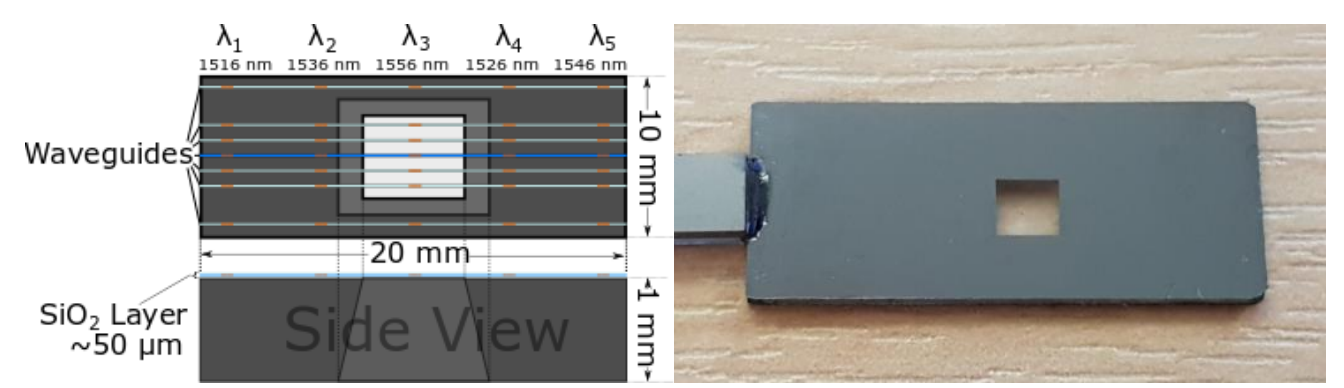

Fig 1. (left) Schematic overview of pressure sensing device including its waveguides and grating locations; (right) top down image of the fabricated device including attached pigtail connector.

\section{Background}

The device was fabricated by growing a $15 \mu \mathrm{m}$ thermal oxide on a $1 \mathrm{~mm}$ thick silicon wafer. Two layers of silicon dioxide was deposited on top of the thermal oxide via flame hydrolysis deposition (FHD). The first deposited layer, the core, has the inclusion of a germanium dopant to increase the photosensitivity of the glass, with boron to ensure the resulting refractive index is matched to the thermal oxide beneath it. The second layer deposited on the top is doped with phosphorus and boron to ensure a lower consolidation point than the core to reduce layer blending during consolidation while again maintaining the zero index contrast. Each layer is consolidated at temperatures around $1200^{\circ} \mathrm{C}$. Once consolidated the wafer is diced into chips and placed in a hydrogen loading cell at $\sim 10{ }^{\circ} \mathrm{C}$ and 120 bar for 7 days to further enhance photosensitivity of the core glass layer.

The creation of the waveguide and gratings were done simultaneously in a single pass using the direct UV writing (DUW) technique [2]. A nominal fluence of $14 \mathrm{~kJ} / \mathrm{cm}^{2}$ was used to write a total of 7 waveguides along the chip with each waveguide having 5 equally spaced Gaussian gratings of $0.5 \mathrm{~mm}$ in length. Gratings were written in the respective order; 1516, 1536, 1556, 1526 and $1546 \mathrm{~nm}$ to minimize the effect of detuning [2]. The refractive index contrast achieved by the DUW was noted to be of the order $10^{-3}$ measured at $1550 \mathrm{~nm}$. 
The fabrication of the diaphragm was achieved by machining the rear surface of the chip to the desired area and the chip was subsequent immersed in tetramethylammonium hydroxide at $70^{\circ} \mathrm{C}$ for 55 hours, yielding a window of dimensions 3.9 by $3.9 \mathrm{~mm}$. Maximum initial diaphragm displacement was $93 \mu \mathrm{m}$ with an average strain of $2.1 \times 10^{3}$ $\mu \varepsilon$ estimated by analysis of the physical profile measured using a Tencor P16 stylus profiler.

For characterization, a polarized broadband source was connected via one arm of a $3 \mathrm{~dB}$ splitter to the waveguide, with the other arm terminated into free space. An optical spectrum analyzer was connected to monitor grating reflections. The diaphragm was placed over a vacuum chuck, the chamber beneath was evacuated and then sealed. The pressure was read using a manual vacuum gauge and the reflection spectrum was recorded. A bleed valve was used to incrementally reduce the differential pressure to characterize over the range of 75 to $0 \mathrm{kPa}$.

\section{Results}

To monitor changes in pressure, each grating was placed into a spectral bin and a Gaussian fit was applied with the fitting parameters recorded. To remove effects of fluctuations in the source intensity, the amplitudes of the grating fit prior to the diaphragm was used as a reference and compared with the grating on the diaphragm. For the grating located on the diaphragm this ratio was then normalized to the point of zero differential pressure producing Fig 2 .

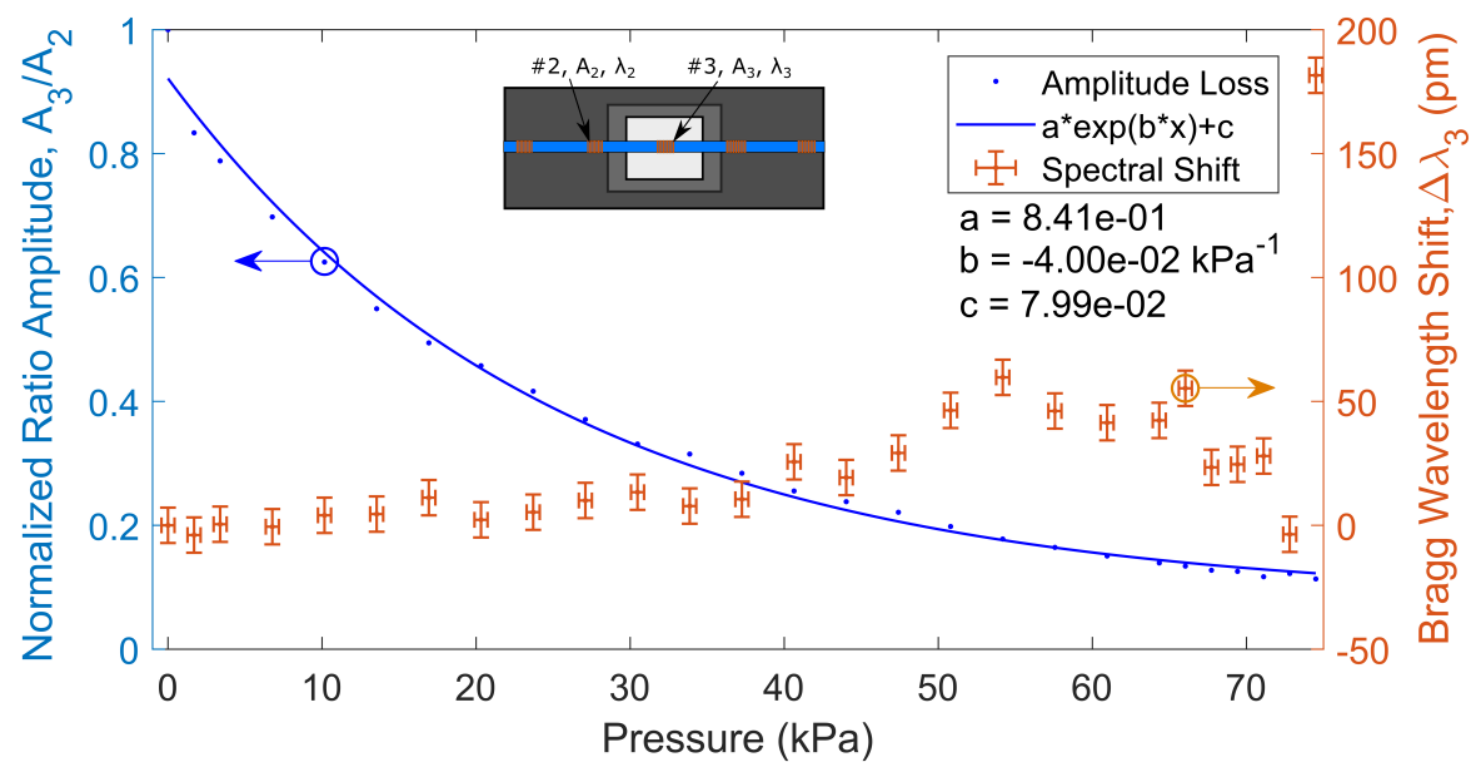

Fig. 2 Left axis shows the amplitude ratio of grating \#2, the reference, and \#3, the measurand, normalized to the zero differential pressure point. Right axis shows the wavelength shift of the \#3 Bragg grating peak with an increase in pressure.

Repeat measurements were made at $10 \mathrm{kPa}$ and a standard deviation of 0.007 in normalized amplitude was obtained. The linear sensitivity between 6.7 and $30.5 \mathrm{kPa}$ was found to be $0.015 \mathrm{kPa}^{-1}$. Combined this yields a resolution of $0.46 \mathrm{kPa}$. The loss due to bending in weakly guiding waveguides is exponential as shown in Fig 2, and this consequently causes a decrease in resolution with an increase in pressure.

\section{Conclusion}

A planar integrated optics approach to monitoring pressure based on ratiometric amplitude loss has been shown. We will present method of fabrication, details on optical behavior, effects of long term jet fuel exposure as well as the latest results on improved device performance, thermal cross sensitivity, stability, full scale resolution and ways in which the device could be made more cost efficient.

\section{References}

[1] Holmes, C., Gates, J. C., \& Smith, P. G. R. (2011). Integrated optical differential pressure transducers achieved using thin buckled silica membranes and direct UV written planar Bragg gratings. Sensors and Actuators, A: Physical, 168(1), 14-21.

[2] Sima, C., Gates, J. C., Rogers, H. L., Mennea, P. L., Holmes, C., Zervas, M. N., \& Smith, P. G. R. (2013). Ultra-wide detuning planar Bragg grating fabrication technique based on direct UV grating writing with electro-optic phase modulation. Optics Express, 21(13), $15747-54$. 